\title{
Application effect of centralized management combined with Information system application management in recycling, cleaning, disinfection and packaging of radiology instruments and reusable medical devices
}

\author{
Xue LIU ${ }^{1,3}$, Yun WANG ${ }^{1,3}$, Wei PAN ${ }^{2,3 *}$
}

\begin{abstract}
To conduct centralized management combined with Information system application management in radiology instruments and reusable medical devices, and explore the promotion value of cleaning quality and work efficiency. The recycling time, cleaning quality, packaging quality and distribution errors of the devices with which centralized management combined with Information system application management (June 2019) was implemented half a year before and after in the radiology department of our hospital were collected and compared. With implementation of centralized management combined with Information system application management, the average recycling time $(25.33 \pm 5.64)$ of devices was remarkably shorter than that before implementation $(42.37 \pm 6.77)(\mathrm{P}<0.05)$. The yield of cleaning $(94.57 \%)$ and packaging $(98.91 \%)$ in the observation group were significantly higher than those in the control group $(86.05 \%)$ and $(87.21 \%)(\mathrm{P}<0.05)$. The probability of distribution error $(8.70 \%)$ and recycling error $(5.43 \%)$ of devices in the observation group were significantly lower than those in the control group $(19.77 \%$ and $18.60 \%)(\mathrm{P}<0.05)$. Centralized management combined with Information system application management effectively improves the cleaning quality and work efficiency of radiology instruments and reusable instruments, indicating long-term application value.
\end{abstract}

Keywords: centralized management; information system application management; radiology instruments; reusable instruments.

Practical Application: Centralized management combined with informationization management effectively improves the cleaning quality and work efficiency of radiology instruments and reusable instruments, indicating long-term application value.

\section{Introduction}

With the development of modern science and technology, the performance and quality of hospital radiology equipment and reusable equipment are getting higher and higher, and the upgrading is becoming faster and faster (Jasmi et al., 2020; Bartlová et al., 2021). However, most domestic equipment management software is for simple operation only, with a lack of equipment dynamic maintenance analysis and processing application, and most of them are only in the development of simple database management application, failing to adapt to the current development of computer hardware technology (Chang et al., 2019; Guo et al., 2015). Therefore, the centralized management of reusable devices is of great significance, which will enhance the clinical treatment quality effectively ${ }^{[1]}$. The application of Information system application management in hospital management maintains obvious advantages, which can effectively improve the consumption of human and material resources in the management process, reduce the complexity of management process, and enhance management effect (Pouls et al., 2020). Therefore, our hospital started to implement centralized management combined with Information system application management in radiology instruments and reusable medical devices since June 2019 and achieved outstanding outcomes. The detailed report is as follows:

\section{Materials and methods}

\subsection{General materials}

The management of radiology instruments and reusable medical devices of our hospital in 2019 was counted and compared, among which, radiology instruments included CT, MRI operation room, scanning room and other reusable instruments, and surgical dilators were investigated. The devices were grouped according to the different management modes.First, the pre-experiment was carried out. The period without centralized management from June to December, 2018 was used as the blank control group, and the period with centralized management from January to June, 2019 was used as the control group. The work efficiency and quality were significantly improved after the implementation of centralized management.The centralized management was conducted only in the devices from January 2019 to June 2019, as the control group, during which radiology instruments and reusable devices were used for a total of 86 times; The centralized management combined with

${ }^{1}$ Department of Radiology Nursing, West China Second University Hospital, Sichuan University/West China School of Nursing, Sichuan University, Chengdu, Sichuan, China ${ }^{2}$ Department of Central Sterile Supply Nursing, West China Second University Hospital, Sichuan University/West China School of Nursing, Sichuan University, Chengdu, Sichuan, China

${ }^{3}$ Key Laboratory of Birth Defects and Related Diseases of Women and Children, Sichuan University, Ministry of Education, Chengdu, Sichuan, China

*Corresponding author: weipan213kh@163.com 
Information system application management was conducted in the devices from June 2019 to December 2019, as the observation group, during which radiology instruments and reusable devices were used for a total of 92 times.During the experimental period, the observation group did not improve other training levels except for training information management, and did not interfere with other measures such as supervision intensity.

\subsection{Methods}

The centralized management was applied to the devices in the control group:

(1) recycling: get to know the conditions of instruments and devices in each department, provide appropriate quantity of instruments for clinical practice, and make preparations for the use of instruments. When recycling, the personnel should take corresponding safeguard measures and use closed vehicles to recycle the devices. In recycling, the devices shall be classified and recycled according to their material composition, shape, size, precision and pollution degree. The number of recycled devices shall be consistent with the number of equipment distributed and used. Place the devices correctly, open the shaft joint and place it in order. should Pay attention to safety and handle with care when taking the devices. Precision instruments shall be placed in the frame of precision instruments for transport, and sharp instruments shall be placed at long intervals to prevent scratches. Catheters and et al. instruments should be cleaned on a special cleaning rack, and the loading capacity should not exceed the standard.

(2) Cleaning: when cleaning the devices, different cleaning methods are adopted according to the recycling classification. Manual cleaning is carried out for devices with high pollution degree, complex structure and precision. The devices used for mechanical cleaning shall meet the requirements for mechanical cleaning, and the setting of cleaning and disinfection procedures should meet relevant specifications for mechanical application. During cleaning, the staff should observe the cleaning process closely, check the operation conditions of the machine and keep records. After the cleaning, strictly observe the aseptic operation and place the cleaned instrument into the drying cabinet for drying. During drying, different temperature and drying time settings for different instruments are also selected according to the classification. The devices, such as puncture needles and catheters, are dried by using a high pressure air gun.

(3) Disinfection: the cleaned devices will be disinfected. The devices being manually cleaned can be disinfected by boiling or adjusting the temperature of drying cabinet. Acidic electrolyzed oxidizing water can also be used to disinfect the devices to reduce the damage to the devices and human body. The devices for mechanical cleaning will disinfected automatically after cleaning.

(4) Packaging: the devices on which the cleaning and disinfection steps are completed shall be packaged. Before packaging, attention should be paid to check whether the equipment is clean and qualified. After visually observing whether the equipment is contaminated, use a magnifying glass for review. In addition to ensuring that the device is bright and stain free, the function of device shall be checked and the catheter should be unobstructed. The device that does not conform to the specification shall be cleaned again in a timely manner and the device with function problems shall be replaced in a timely manner. The packaging shall be carried out in the aseptic condition, and two layers of packaging materials shall be used for closed packaging after inspection. One layer of packing may be used for sealed packing. Upon completed packaging, the type and quantity of devices and the pre-packaging inspection results shall be clearly indicated on the outside of the package. After that, the packaged device will be stored on a shelf at a suitable temperature and environment for use.

Information system application management was added to the observation group based on this: (1) organize personnel training before application of Information system application management system to ensure that all personnel involved in the using, recycling, cleaning, disinfection, packaging and other processes understand and become skilled in using the information management system, and master the standard operation mode. (2) after confirming the instruments and devices needed for treatment, each department will initiate an application on the system, and make clear the quantity and type of instruments needed so that the disinfection supply room can release them as needed. Perform classification operations strictly from device release to recycling, define the type and quantity of devices used in the department, log in the system in time after use and select the "RECYLCE" option to recycle the device in time, meanwhile, compare and check whether the devices to be recycled are consistent with those being distributed, and the inspection results can be uploaded to the system through photos and confirmation of person in-charge. (3) after receiving the notification of device recycling, the disinfection supply room shall perform device recycling in a timely manner, and make explanations if the time between request initiation and confirmation of recycling exceeds a reasonable time. When recycling, classified recycling shall be carried out according to the release records recorded in the system, and timely cause tracing shall be carried out for devices that do not conform to the system information. The devices of the same batch of recycling shall be recorded according to the classification difference and the recycling equipment department. Before proceeding to the next procedure, the devices state shall be confirmed to be consistent with the system record of the previous procedure and the problems shall be found and declared in time. (4) the barcode of devices formed after disinfection will generate the state of disinfected devices and relevant parameters executed in disinfection. The device disinfection state can be confirmed by scanning the barcode when accessing the device package (Figure 1).

\subsection{Observation index}

The difference in recycling time of devices in the two groups was compared according to the end time of device using and the time of devices recycled in the disinfection supply room. 
The yield of cleaning and packaging of devices was compared based on relevant result of inspection by using the recycled devices.

The distribution errors and classification errors after recycling were compared between the two groups.

\subsection{Statistical analysis}

SPSS21.0 data analysis software was used for data analysis, measurement data were represented by $\left({ }^{-} \mathrm{x} \pm \mathrm{s}\right)$ and " $\mathrm{t}$ " test was used for inspection; count data were represented by (\%) and inspected using Chi-square. The comparison result $(\mathrm{P}<0.05)$ indicated the difference was statistically significant.

\section{Results}

\subsection{Comparison of difference in recycling time of devices in both groups}

With implementation of centralized management combined with Information system application management, the average recycling time $(25.33 \pm 5.64)$ of devices was remarkably shorter than that before implementation $(42.37 \pm 6.77)$ and the comparison between both groups was statistically significant $(\mathrm{P}<0.05)$, as shown in Table 1.

\subsection{Comparison of difference in cleaning and packaging of devices in both groups}

The yield of cleaning (94.57\%) and packaging (98.91\%) in the observation group were significantly higher than those in

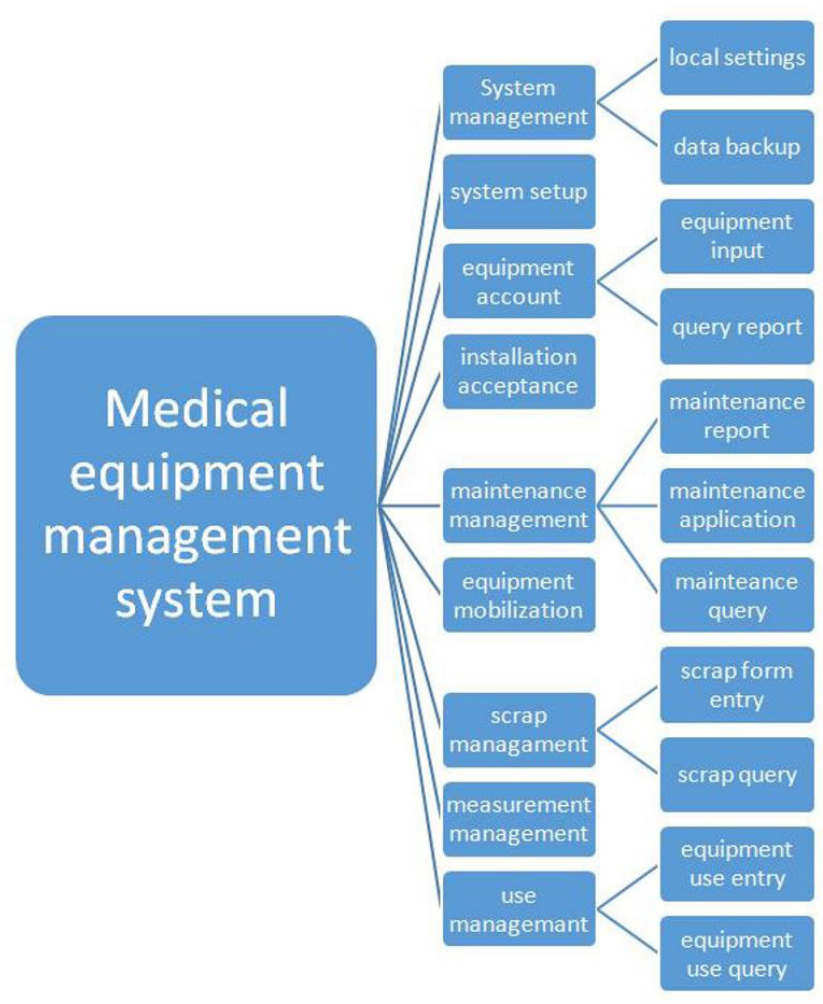

Figure 1. Information system application management System of Medical Devices. the control group (86.05\%) and (87.21\%), and the comparison between both groups was statistically significant $(\mathrm{P}<0.05)$, as shown in Table 2.

\subsection{Difference in distribution error and recycling error}

The probability of distribution error $(8.70 \%)$ and recycling error $(5.43 \%)$ of devices in the observation group were greatly lower than those in the control group (19.77\%) and (18.60\%), and the comparison between both groups was statistically significant $(\mathrm{P}<0.05)$, as shown in Table 3.

\section{Discussion}

The reusable instruments are more and more widely used in modern clinical work, to reduce financial pressure for hospitals and increase the utilization efficiency of medical resources. Therefore, the basic management of devices, and safety management of production equipment are improved clinically day by day. Radiology instruments and reusable instruments are usually used for many times, which is not only relieves the financial pressure of hospitals, but also increases the application efficiency of medical resources. Due to its repeatability and strong mobility among patients, it plays a key role in instrument cleaning, device recycling, cleaning, disinfection and packaging, to ensure the safety of devices, reduce the risk of iatrogenic injuries in treatment, and reduce nurse-patient disputes (Clevert et al., 2004).

It is pointed out in WS-3101-2016 central disinfection and supply department (CSSD). Part I: management standards that

Table 1. Comparison of difference in recycling time of devices in both groups $[(-\mathrm{x} \pm \mathrm{s}), \mathrm{min}]$.

\begin{tabular}{cc}
\hline Group & Recycling Time \\
\hline Observation group $(\mathrm{n}=92)$ & $25.33 \pm 5.64$ \\
Control group $(\mathrm{n}=86)$ & $42.37 \pm 6.77$ \\
$t$ & 18.290 \\
$P$ & 0.000 \\
\hline
\end{tabular}

Table 2. Comparison of difference in cleaning and packaging of devices in both groups $[n,(\%)]$.

\begin{tabular}{ccc}
\hline Group & Yield of Cleaning & Yield of Packaging \\
\hline Observation group $(\mathrm{n}=92)$ & $87(94.57)$ & $91(98.91)$ \\
Control group $(\mathrm{n}=86)$ & $74(86.05)$ & $75(87.21)$ \\
$x^{2}$ & 4.148 & 10.598 \\
$P$ & 0.042 & 0.001 \\
\hline
\end{tabular}

Table 3. Comparison of difference in distribution error and recycling error [n, (\%) ].

\begin{tabular}{ccc}
\hline Group & Distribution Error & Recycling Error \\
\hline Observation group $(\mathrm{n}=92)$ & $8(8.70)$ & $5(5.43)$ \\
Control group $(\mathrm{n}=86)$ & $17(19.77)$ & $16(18.60)$ \\
$x^{2}$ & 5.019 & 8.204 \\
$P$ & 0.025 & 0.004 \\
\hline
\end{tabular}


the work of disinfection supply centers has a direct impact on the quality and safety of medical care, and the management of their work should be part of the management of infections. Meanwhile, it's suggested in relevant requirements that the procedures such as routine maintenance of equipment and device recycling shall be traceable (Wray et al., 2018; Hu et al., 2020). There're no unified and standard titles of instrument, equipment origin, model, maintenance cost, et al., for many domestic devices, which is often in a non-scientific state without standard, and maintenance and maintenance are also chaotic. Information sharing cannot be formed among various units, and the superiority of information technology cannot be given full play. Centralized management has achieved resource integration to a large extent and reduced the difficulty of reasonable allocation of medical resources (Zaloga et al., 2019; Schenk et al., 2020). The centralized management of multiplexed devices implemented in our hospital according to relevant specifications has effectively improved some problems and achieved good outcomes. The centralized management of multiplexed devices implemented in our hospital according to relevant specifications has effectively improved some problems and achieved good results. However, with the continuous progress of work, it is found that its shortcomings are more and more distinct (Wang \& Wei, 2019; Choeisoongnern et al., 2020). The centralized management involves a lot of contents and objects, and the work is complex, especially for the reusable equipment, there is usually a more complex cleaning, disinfection and inspection procedure between the recycle and reuse. To define clear responsibility, a large number of forms may need to be filled, which not only increases the difficulty of the work, but also makes the responsibility traceability harder when the data is accumulated (Conti \& Orcioni, 2019; Wen et al., 2019). In addition, centralized management can only master the situation of device before use and after recycle. It is difficult to track the device dynamically after release, and understand the demand of devices and recycle request, which greatly reduces the difficulty of work and increases the consumption of human and material resources. Informationization has been widely applied in modern medical treatment, but its application in hospital management practice is still limited (Qin et al., 2018; Yu et al., 2018).

Information system application management is based on the big data platform. It has a comprehensive data collection system, and can retrieve the required information more accurately and quickly. It is easier to implement the responsibility, and record the responsibility information more comprehensively and search as soon as possible (Ahmadi et al., 2018). Moreover, Information system application management can give full play to the advantages of resource sharing and information communication to a larger extent, track the device dynamics, timely understand the detailed information of device requirements and grasp the recycle demand as soon as possible, greatly reducing the time spent on recycle. Meanwhile, by virtue of the system, Information system application management can further optimize the recycling, cleaning, disinfection, packaging and other procedures of reusable devices, conduct strict specifications for disinfection of devices, ensure the implementation of all procedures, strengthen the supervision mechanism, make it easier to trace the source of responsibility management, solve problems as soon as possible, and reduce the risk of adverse events once again. It can not only effectively reduce the occurrence of adverse events and disqualification, but also reduce manpower demand, improve communication efficiency, and greatly improve the work efficiency of hospital staff. Besides, it shall be defined clearly in hospital management that the intervention of Information system application management into mature application is not a process that can be accomplished overnight, therefore, it is necessary to gradually collect relevant information inside and outside the hospital, constantly improve the information of relevant management devices, and find problems and solve them in the application process, so as to improve the application value of Information system application management.

We will lay the foundation for the service life cycle and service cost of devices through Information system application management. By introducing advanced business process dynamic management technology, the instrument management process can be optimized effectively. Meanwhile, the model of fault analysis and preventive maintenance can be set up, for reasonable evaluation of scientific management of inventory, demand analysis and inventory early warning. Perfect information management will effectively establish all kinds of data collection, processing and analysis, provide data support and data analysis support for management decision, and support continuous improvement of management process. In the process of hospital management, clear intervention of Information system application management is not a process accomplished in an action, instead, it needs collection of related information inside and outside the hospital, continuous perfection of improve relevant information of management equipment, and unceasingly discovery and solution of problems during application, so as to improve the application value of Information system application management.

In conclusion, centralized management combined with Information system application management will effectively improve the cleaning quality of radiology instruments and reusable devices, effectively control the unqualified conditions of devices being used, improve work efficiency, and reduce doctor-patient disputes, maintaining long-term application value. The attention should be paid to the training on relevant personnel during application, and the management and operation standards should be grasped strictly, so as to improve the application benefits of Information system application management.

\section{Conflict of interest}

The author declare that they have no conflict of interest.

\section{Fundings}

The authors declare that no financial support was received.

\section{Author contributions}

Xue Liu and Wei Pan carried out study concepts and design, definition of intellectual content and was the guarantor of integrity of the entire study. Wei Pan and Yun Wang contributed to clinical studies and experimental studies. Xue Liu, Yun Wang and Wei 
Pan contributed to data acquisition and analysis, manuscript preparation, editing and review.

\section{References}

Ahmadi, E., Masel, D. T., Metcalf, A. Y., \& Schuller, K. (2018). Inventory management of surgical supplies and sterile instruments in hospitals: a literature review. Health Systems, 8(2), 134-151. http://dx.doi.org/ 10.1080/20476965.2018.1496875. PMid:31275574.

Bartlová, M., Ziółkowska, D., Pospiech, M., Shyichuk, A., \& Tremlová, B. (2021). Determination of carrageenan in jellies with new methylene blue dye using spectrophotometry, smartphone-based colorimetry and spectrophotometric titration. Food Science and Technology, 41(Suppl. 1), 81-90. http://dx.doi.org/10.1590/fst.01220.

Chang, C. W. D., Brenner, M. J., Shuman, E. K., \& Kokoska, M. S. (2019). Reprocessing standards for medical devices and equipment in otolaryngology: safe practices for scopes, speculums, and single-use devices. Otolaryngologic Clinics of North America, 52(1), 173-183. http://dx.doi.org/10.1016/j.otc.2018.08.014. PMid:30262168.

Choeisoongnern, T., Sivamaruthi, B. S., Sirilun, S., Peerajan, S., Choiset, Y., Rabesona, H., Haertlé, T., \& Chaiyasut, C. (2020). Screening and identification of bacteriocin-like inhibitory substances producing lactic acid bacteria from fermented products. Food Science and Technology, 40(3), 571-579. http://dx.doi.org/10.1590/fst.13219.

Clevert, D. A., Jung, E. M., Reiser, M., \& Rupp, N. (2004). Materials in interventional radiology-computer program. Lofoy, 176(10), $1475-1480$.

Conti, M., \& Orcioni, S. (2019). Cloud-based sustainable management of electrical and electronic equipment from production to end-of-life. International Journal of Quality \& Reliability Management, 36(1), 98-119. http://dx.doi.org/10.1108/IJQRM-02-2018-0055.

Guo, F., Xie, Y., Li, S., Lata, J., Ren, L., Mao, Z., Ren, B., Wu, M., Ozcelik, A., \& Huang, T. J. (2015). Reusable acoustic tweezers for disposable devices. Lab on a Chip, 15(24), 4517-4523. http://dx.doi.org/10.1039/ C5LC01049G. PMid:26507411.

Hu, C., Wang, X., \& Wang, Y. H. (2020). Research on equipment management system based on robot laboratory. Journal of Computer and Communications, 8(07), 23-31. http://dx.doi.org/10.4236/ jcc.2020.87003.

Jasmi, N., Mansor, N., Lim, E. J., Yusof, N. L., Hajar-Azhari, S., \& Rahim, M. H. A. (2020). The effect of sonication and heat treatment on the physicochemical, nutritional and microbiological properties of different sugarcane variants. Food Science and Technology, 40(3), 551-556. http://dx.doi.org/10.1590/fst.12619.

Pouls, B. P. H., Kristensen, L. E., Petersson, M., Van Den Bemt, B. J. F., Ballerini, L., Bruggraber, R., Karlen, H., Mountian, I., Van Bracht, E., Wiegratz, S., \& Jørgensen, T. S. (2020). A pilot study examining patient preference and satisfaction for ava ${ }^{\oplus}$, a reusable electronic injection device to administer certolizumab pegol. Expert Opinion on Drug Delivery, 17(5), 705-711. http://dx.doi.org/10.1080/17425 247.2020.1736552. PMid:32154740.

Qin, X. W., Jiang, Z. Z., Ip, W. H., Sheng, Y., \& Wu, C. H. (2018). Analyzing manufacturer and the insurance-based risk mitigation policy with equipment service contracting. Enterprise Information Systems, 12(10), 1359-1381. http://dx.doi.org/10.1080/17517575.2 018.1527043 .

Schenk, L., Feychting, K., Annas, A., \& Öberg, M. (2020). Calls made to the poisons information centre reveal need for improved risk management of cleaning agents in the workplace. International Journal of Occupational Safety and Ergonomics, 26(1), 140-148. http://dx.doi.org/10.1080/10803548.2018.1550297. PMid:30451643.

Wang, Y. T., \& Wei, Z. H. (2019). Research and Implementation of Big Data Technology Laboratory Equipment Reservation Management System. IOP Conference Series: Earth and Environmental Science, 252, 042072 .

Wen, M., Lu, B., Li, S., \& Kang, R. (2019). Location and allocation problem for spare parts depots on integrated Iogistics support. Journal of Systems Engineering and Electronics, 30(6), 1252-1259. http://dx.doi.org/10.21629/JSEE.2019.06.19. [English version]

Wray, S., Hayward, B., Dangond, F., \& Singer, B. (2018). Ease of use of two autoinjectors in patients with multiple sclerosis treated with interferon beta-1a subcutaneously three times weekly: results of the randomized, crossover REDEFINE study. Expert Opinion on Drug Delivery, 15(2), 127-135. http://dx.doi.org/10.1080/1742524 7.2018.1407755. PMid:29206056

Yu, K. J., Zhou, X. J., \& Luo, S. (2018). Design of hospital scientific research talent management information software system. Journal of Discrete Mathematical Sciences and Cryptography, 21(6), 1461-1466. http://dx.doi.org/10.1080/09720529.2018.1527815.

Zaloga, V., Dyadyura, K., Rybalka, I., \& Pandova, I. (2019). Implementation of integrated management system in order to enhance equipment efficiency. Management Systems in Production Engineering, 27(4), 221-226. http://dx.doi.org/10.1515/mspe-2019-0035. 